The Jackson Laboratory

The Mouseion at the JAXlibrary

Faculty Research 2021

Faculty Research

$10-1-2021$

The Jackson Laboratory Nathan Shock Center: impact of genetic diversity on aging.

Ron Korstanje

Luanne L. Peters

Laura Robinson

Stephen D Krasinski

Gary Churchill

Follow this and additional works at: https://mouseion.jax.org/stfb2021

Part of the Life Sciences Commons, and the Medicine and Health Sciences Commons 


\title{
The Jackson Laboratory Nathan Shock Center: impact of genetic diversity on aging
}

\author{
Ron Korstanje (D) Luanne L. Peters (D) \\ Laura L. Robinson • Stephen D. Krasinski • \\ Gary A. Churchill
}

Received: 7 July 2021 / Accepted: 11 July 2021 / Published online: 23 July 2021

(C) American Aging Association 2021

\begin{abstract}
Healthspan is a complex trait, influenced by many genes and environmental factors that accelerate or delay aging, reduce or increase disease risk, and extend or reduce lifespan. Thus, assessing the role of genetic variation in aging requires an experimental strategy capable of modeling the genetic and biological complexity of human populations. The goal of the The Jackson Laboratory Nathan Shock Center (JAX NSC) is to provide research resources and training for geroscience investigators that seek to understand the role of genetics and genetic diversity on the fundamental process of aging and diseases of human aging using the laboratory mouse as a model system. The JAX NSC has available novel, deeply characterized populations of aged mice, performs state-of-the-art phenotyping of age-relevant traits, provides systems genetics analysis of complex data sets, and provides all of these resources to the geroscience community. The aged animal resources, phenotyping capacity, and genetic expertise available through the JAX NSC benefit the geroscience community by fostering cuttingedge, novel lines of research that otherwise would not be possible. Over the past 15 years, the JAX NSC has transformed aging research across the geroscience community, providing aging mouse resources and tissues to researchers. All JAX NSC data and tools are
\end{abstract}

R. Korstanje $(\bowtie) \cdot$ L. L. Peters · L. L. Robinson ·

S. D. Krasinski · G. A. Churchill

The Jackson Laboratory, Bar Harbor, ME, USA

e-mail: ron.korstanje@jax.org publicly disseminated on the Mouse Phenome Database and the JAX NSC website, thus ensuring that the resources generated and expertise acquired through the Center are readily available to the aging research community. The JAX NSC will continue to enhance its ability to perform innovative research using a mammalian model to illuminate novel genotype-phenotype relationships and provide a rational basis for designing effective risk assessments and therapeutic interventions to boost longevity and disease-free healthspan.

Keywords Healthspan - Lifespan - Genetic diversity $\cdot$ Complex trait $\cdot$ Diversity outbred mice

\section{Introduction}

Extraordinary improvements in environmental conditions and advances in medical care have led to significant extension of the average human lifespan and improved health in later life. However, aging is still associated with declining function in virtually every organ system. Lifespan and disease-free healthspan are intricately entwined complex traits influenced by genetic factors that modulate the aging process and age-related disease risk. Studies of long-lived individuals and families estimate that $15-25 \%$ of the variation in human lifespan is determined by genetic factors [1-3]. Genome-wide association studies confirm that many genes individually exert small effects that 
influence human aging, and that the effects of these genes are modified by environmental factors $[4,5]$. To model the genetic and biological complexity of humans and human aging phenotypes, a genetically tractable experimental organism, maintained in a controlled and easily manipulated environment, is necessary for us to significantly advance aging research. Mice are an ideal mammalian model for studying the genetics of aging because their genomes are wellcharacterized, their environment can be easily controlled, their lifespan is relatively short, and considerable resources are readily available.

The goal of the The Jackson Laboratory Nathan Shock Center (JAX NSC) is to provide research resources and training for geroscience investigators that seek to understand the role of genetics and genetic diversity on the fundamental process of aging and diseases of human aging using the laboratory mouse as a model system. The JAX NSC has available novel, deeply characterized populations of aged mice, performs state-of-the-art phenotyping of agerelevant traits, provides systems genetics analysis of complex data sets, and provides these resources to the geroscience community. The Center provides phenotyping expertise, capacity, and space for investigators to develop their own interventions and phenotyping assays. The aged mouse resources, phenotyping capacity, and genetic expertise available through the JAX NSC benefit the geroscience community by fostering cutting-edge, novel lines of research that otherwise would not be possible. The Center will continue to enhance the ability to perform innovative research using a mammalian model that can illuminate novel genotype-phenotype relationships and provide a rational basis for designing effective risk assessments and therapeutic interventions to boost longevity and disease-free healthspan.

Many of the studies supported by the JAX NSC utilize the Collaborative Cross (CC) [6, 7], and their eight parental (founder) strains (Fig. 1). The CC mice were generated and maintained as genetically diverse recombinant inbred lines, and therefore, each locus is homozygous for a particular founder strain allele. Since CC are inbred, each strain can be maintained and replicated for further study. The Diversity Outbred (DO) mice, derived from the same founder strains, are generated as an outbred population, and as such are highly heterozygous $(\sim 87.5 \%$ of the genome) - this high level of heterozygosity genomewide is similar to what is observed in human populations. Continuous outbreeding ensures that each DO mouse is genetically unique.
Fig. 1 Generation of Diversity Outbred (DO) and Collaborative Cross (CC) mice

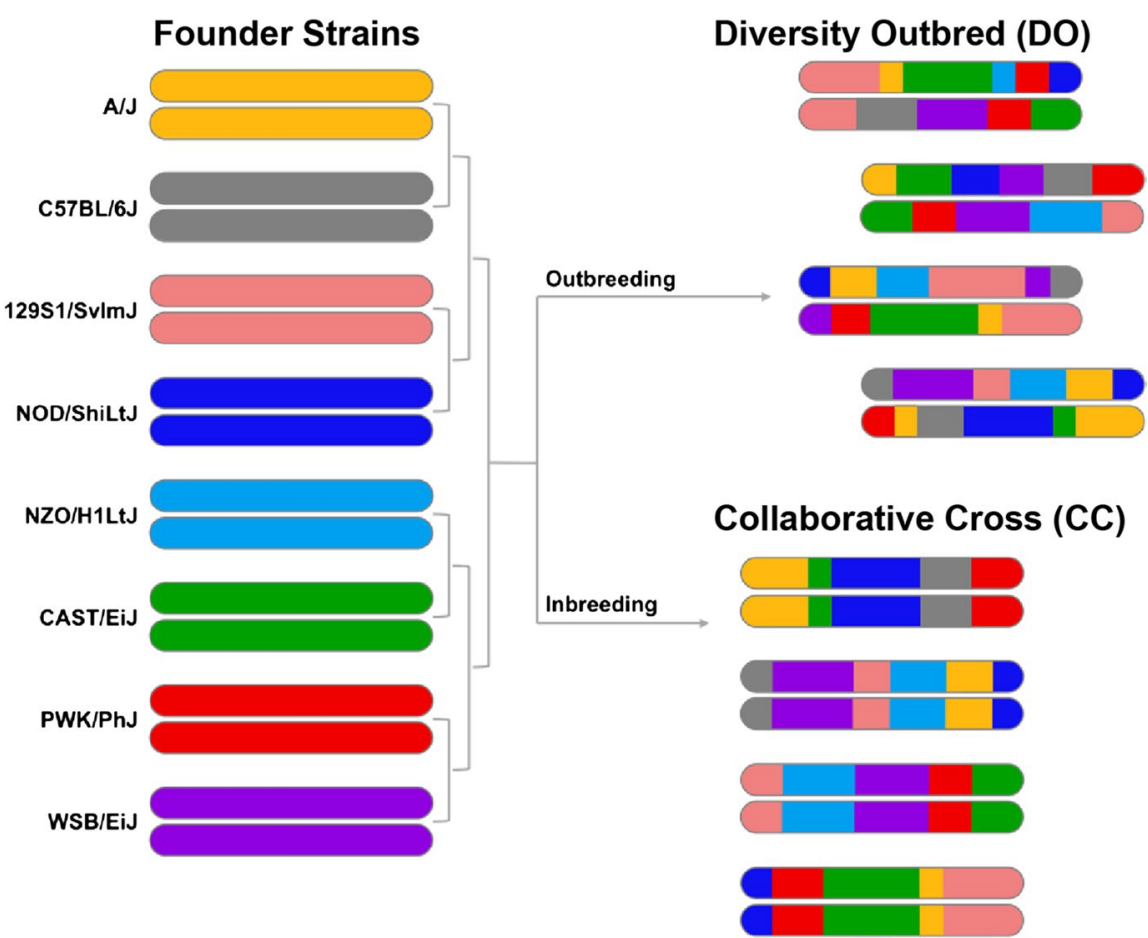


In addition to the genetic diversity in the nuclear genome, the founders show significant differences in the DNA sequences of their mitochondrial genome, in which differences in haplotype have been linked to the aging process [8]. These differences can be exploited in founders and their CC and DO offspring to study the impact of genetic diversity in mitochondrial DNA on aging.

The complementary $\mathrm{CC}$ and DO mice provide unique genetically diverse resources to comprehensively test the influence of host genetic background on aging and age-related phenotypes. Previous studies have demonstrated that nearly every measured age-related phenotype, including molecular (e.g., gene expression levels), physiological (e.g., frailty), and behavioral (e.g., memory) traits, is highly variable in CC and DO mice, and that this variability is largely due to standing genetic variation in the population [9]. These studies provide strong support for the scientific premise that the DO and CC mice are optimal models for studying the impact of genetic variability on aging.

The JAX NSC is a one-stop-shop for investigators who want to conduct age-related research using mice but lack the resources or experience to initiate an aging mouse experiment. Access to aged mice and the cost of housing animals represent significant barriers to entry into this field. Researchers facing these challenges can initiate new projects through our Research Development Core, which utilizes an innovative in-kind program where experiments for Pilot Program awardees are coordinated and streamlined, enabling the Center to perform more experiments for more investigators by making efficient use of the resources that are available through the JAX NSC. From 2017 to 2020, 38 different investigators have performed aging experiments. Our rolling enrollment colony of aging mice provides the animals needed for these experiments and is managed by the Animal and Phenotyping Core. This aged animal resource includes not only the always popular C57BL/6 J strain, but also the $\mathrm{CC}$ and other inbred strains, the DO population, and genetically engineered mice. The Core maintains the facilities and a highly skilled team of technicians to carry out sophisticated phenotyping assays optimized for aged mice [10,11]. These assays include advanced analytical techniques that use artificial intelligence and machine learning (e.g., video analysis of gait and behavior, continuous monitoring metabolic cages, and image analysis of pathology offered in the Image Analysis Core). JAX NSC staff are adept at learning new techniques. However, some investigators require hands-on access to animals to conduct their research. Accordingly, the JAX NSC offers a Visiting Scientist Program for investigators who want to participate directly in their experiments. The Statistics and Data Analysis Core provides cloudbased data management services and assistance with data analysis. Investigators can be involved in their study at any level, from simply providing an experimental design and having JAX NSC staff perform the study, collect tissues, and analyze data, to spending time onsite to oversee and carry out the experimental work in Center facilities. Much of this can be accomplished at no cost to investigators, with support provided through the Pilot Program. JAX NSC scientists work closely and follow up with Pilot Program awardees to ensure that they have the long-term support they need to publish their findings and/or apply for additional NIH funding to continue their research.

\section{Research cores}

\section{Research Development Core}

Aging research is challenging because aging studies are both lengthy and expensive. Most research grants do not include funds to develop aging resources, and there is limited access to the resources required to successfully compete for external funding in the aging field. The lack of access to aging resources can dissuade investigators from engaging in aging research. Facilitating and sustaining young scientists who wish to establish an independent career in aging research, or established scientists looking to make a career turn into the field of aging, is essential for expanding the field and ensuring a critical mass of aging researchers for the future. A mechanism for pilot awards is crucial to enable exploration of new ideas and to obtain preliminary data that is essential to obtain independent research funding.

The mission of the JAX NSC is to provide access to diverse resources (e.g., aged mice, phenotype data, omics data, tissues from aged mice, training in aging biology) that cannot be generated or funded by 
individual labs. By providing a variety of computational, intellectual, and genetic resources, the JAX NSC enables investigators to initiate aging studies efficiently and effectively.

The primary mechanism for outreach is our Pilot Project Program, which provides in-kind services and access to our core resources (described below). Providing these pilot awards and resources in an environment that offers training, mentorship, and career opportunities ensures the successful development and/or transition of new investigators into aging research. An annual request for applications is circulated through the Nathan Shock Centers Coordinating Center (nathanshockcenters.org). Investigators are asked to submit a two-page proposal describing the aims and rationale for their project. Project proposals are then reviewed and ranked by JAX NSC faculty, according to the following criteria:

- Will the funds support a new investigator or help an established investigator transition into aging research?

- What is the likelihood that the work will lead to a successful grant application?

- Will the project leverage or enhance JAX NSC resources?

Once awards are approved, members of JAX NSC staff contact the investigators to plan and discuss the details of the experiments. In the event the awardee is directly involved in the experimental techniques, arrangements are made to host the awardee and provide access to the needed facilities. When possible, experiments from different investigators will be combined to make optimal use of the limited number of aged mice. Since moving to our new in-kind format in 2017, in which we perform experiments using aged mice at the request of investigators, 38 pilot projects have been awarded (see agingmice.jax.org for an overview of projects).

\section{Animal and Phenotyping Core}

Aging studies are lengthy and expensive, and access to unique animal resources and state-of-the-art phenotyping capabilities is limited. The mission of the Animal and Phenotyping Core is to maintain genetically diverse populations of aged mice and provide state-of-the-art phenotyping services for age-relevant traits to support pilot projects and internal studies devoted to aging research. The Core provides support for short- and long-term studies using cross-sectional and longitudinal study designs and has generated data for many studies over the years, such as the identification of Lhfp as a regulator of bone mass [12] and Dlgap2 as a regulator of age-related cognitive decline and Alzheimer's dementia [13], detection of strain and sex differences in IGF1 levels [14], and determination of a multi-tissue full lifespan epigenetic clock for mice [15].

The Animal and Phenotyping Core maintains cohorts of aged mice, including the inbred strain C57BL/6 J, the historical workhorse of aging research for which a huge body of lifespan and healthspan data exists. To promote the use of genetic diversity in aging studies, we currently maintain cohorts of aged DO mice. Aged mice cohorts are populated using a rolling enrollment strategy to ensure that mice at young (6-8 months), middle (12-15 months), and old (18-24 months) ages are available at the same time to support cross-sectional studies. The Core also has aged CC inbred strains, F1 hybrid mice, and transgenic knockout mouse models for specific studies. For example, we implemented an intermittent fasting protocol with cohorts of CC mice on 2-day/week fasting or ad libitum control diets using both longitudinal and cross-sectional cohorts of $10 \mathrm{CC}$ strains, a total of 800 mice in 1:1 sex ratio. The phenotypic analysis of genetically diverse aging mice on dietary interventions will help us to understand how genetic background interacts with interventions to shape effects on longevity. This is but one of several experiments to collect genetic and phenotypic data on aged animals under controlled dietary interventions. The cumulative data will enhance the value of the DO and CC mice as resources for aging research.

The Animal and Phenotyping Core staff have developed and refined phenotyping methods over many years. The breadth and quality of these techniques reflect the Center's collective experience in assessing the health of aged mice. Longitudinal studies are carried out with comprehensive and noninvasive phenotyping methods. The phenotyping protocols developed and validated by the JAX NSC are described on the JAX NSC website (agingmice. jax.org), the Mouse Phenome Database (MPD) (phenome.jax.org), and elsewhere [10, 16]. Standard offerings include a comprehensive suite of clinical 
healthspan evaluations: frailty index, grip strength, rotorod, wheel running, acoustic startle, complete blood counts, immune function, glucose tolerance tests, urinary testing to monitor kidney function, echocardiography, and body composition including bone mineral density and fat content using advanced imaging modalities. The adjacent Jackson Laboratory Center for Biometric Analysis (CBA) houses dedicated facilities and trained staff to conduct a wide range of behavioral testing, including Y maze, tail suspension, novel object recognition, light/dark box, and open field for activity- and anxiety-related behaviors. Our Core phenotyping technicians are trained and rigorously evaluated before being certified to carry out these tests. In addition to phenotyping, we collect tissues, blood, urine, and fecal samples for molecular profiling and microbiome studies. For example, in one study, we used the kidneys of DO mice collected at 6,12 , and 18 months of age to better understand the molecular changes that take place in the kidney during the aging process and measured both mRNA levels and protein levels. We found that proteins show a greater extent of change than mRNA, which suggests that mRNA profiling alone provides an incomplete picture of molecular aging and that examination of changes in proteins is essential to understand aging processes that are not transcriptionally regulated [17]. Tissues and blood samples are also being used to confirm findings in other (human) studies. For example, blood samples collected in our Core were used to test conservation and association with lifespan of circRNAs that were associated with human aging phenotypes [18].

Our phenotyping capabilities are continuously evolving to meet the needs of the scientific community. The Core helped to develop a novel urinary bladder function assay [19] that is now a routine assay. Promethion metabolic cages (Sable Systems) were incorporated into the phenotyping suite, which has enabled us to evaluate energy expenditure, metabolic substrate selection, food and water uptake, meal and drinking patterns, position, total activity, and wheel running. Most recently, we have worked with JAX scientists to develop and evaluate a videobased testing paradigm coupled with novel machine learning to monitor activity, specific behaviors, gait function, and frailty in aging mice. We anticipate that these video-based techniques will support large-scale phenotyping while minimizing handling stress on aged animals. These and other new techniques allow the JAX NSC to assess the complex phenotypes that are relevant to aging humans in today's society.

Phenotyping of very old mice requires extreme care, and many procedures including anesthesia or blood collection are often not feasible in the oldest animals ( $>2.5$ years). We have developed a novel "fragility index" that expands the standard mouse frailty index [20] to evaluate the health of mice that are nearing the end of life. Animals in the Core facilities are subject to frequent welfare checks and monitoring. We work closely with our Institutional Animal Care and Use Committee to ensure humane treatment of all animals, but especially for animals in late life.

The Animal and Phenotyping Core will design and implement custom phenotyping pipelines to meet the needs of the investigator. We have implemented longitudinal studies that include lifespan as an end point or require non-invasive phenotyping. Cross-sectional studies are typically designed to collect tissues and plasma or to evaluate the short-term response to interventions. We can deliver compounds to animals in food or water. For example, we have used drinking water to administer methylene blue, and 4-phenylbutyrate, and have developed diets to administer rapamycin, mianserin, and 3-hydroxyanthranilic acid in food. These include evaluation of food and water intake to ensure proper dosing. One example is a recently published study on oral rejuvenation in which we formulated a specialized diet containing rapamycin and, at the end of the treatment period, used $3 \mathrm{D}$ imaging to examine the bone around teeth [21].

A key element to the success of our Animal and Phenotyping Core is our ability to maximize the use of aged animals by integrating experiments. We can successfully accommodate phenotyping requests from multiple investigators. The use of the same animals across multiple projects, when feasible, provides economy and expands our capacity to deliver more investigator-initiated projects. Attention to the order in which the procedures are performed ensures that any given measure is not adversely influenced by preceding tests. For example, if behavioral testing is requested by a researcher, the Core would perform these assays at the beginning of the protocol, shifting other testing or interventions forward or otherwise rearranging the schedule to accommodate 
multiple requests. In this way, the effects of stress on the behavioral assays are minimized.

Data collection and quality control (QC) begin in the Animal and Phenotyping Core. QC is a key component of day-to-day operations. Many data types, e.g., body weights on electronic scales, are collected directly into our Laboratory Information Management System (LIMS) without the need for intermediate data recording and transfer. Our LIMS also supports daily review of data by technicians. With new phenotyping technologies constantly being developed, the scope of data collection and analysis continues to grow. Thus, a well-functioning and efficient LIMS is essential to track animals, schedule testing, and provide a stable repository for primary data collection. During peak periods, the Core may collect thousands of data points each week. We employ a highly effective technique to reduce animal mix-ups: at the start of each phenotyping procedure, technicians record body weight, coat color, and ear notch identification; these are required data entry fields that are not auto-completed. Comparison of the recorded values to expected values is sufficient to catch the majority of mix-up events. Data generated by the Animal and Phenotyping Core is migrated to the JAX NSC Data and Statistical Core for further QC and analysis. Close communication between the data scientists and phenotyping technicians is key to ensuring robust and high-quality data for downstream analysis.

\section{Data and Statistical Core}

Mouse studies sponsored by the JAX NSC produce an enormous amount of data, including phenotypic, genetic, epigenetic, expression profiling, and microbiome profiling data. The focus of the JAX NSC on genetic diversity using $\mathrm{CC}$ inbred and DO mice requires the analysis of hundreds of mice. With new phenotyping technologies constantly being developed, the scope of data collection and analysis continues to grow. Thus, a well-functioning and efficient data management and statistical analysis operation is necessary.

The Data and Statistical Core is responsible for the management, quality control, analysis, and dissemination of data for all JAX NSC projects. Improved data management and quality control methods, outreach to promote use of JAX NSC data, and engagement with pilot project investigators accelerate the pace of aging research and increase the accessibility of JAX NSC data and other resources. Our CLIMB LIMS (Rockstep Inc., Portland ME) provides the integrated data management and workflow scheduling processes.

Data QC is a time-consuming process. It includes cross-checking husbandry and task-scheduling data against the raw data records. Most data errors are corrected directly in the LIMS, which then becomes the definitive source of cleaned raw data - version control ensures that all changes are traceable. Many data types require additional process steps, including batch correction and standardization prior to analysis. These steps are done outside the LIMS environment and are supported by reproducible documentation, in the form R, python or shell scripts. Upon completion of these pre-processing steps, data is delivered to investigators, migrated to the JAX NSC website, or submitted to the MPD [22].

The primary activity of MPD is to collect mouse strain phenotype datasets where mice are tested following a defined protocol. The MPD was recently updated specifically to enhance its utility in producing aging data as part of the JAX NSC resource initiative. New tools to handle time-course data and improve Kaplan-Meier survival curves were developed to advance the ability of investigators to identify correlations across strains, phenotypes, and datasets, and to use JAX NSC data in their research. To support aging research, MPD researchers also developed new interfaces for survival, pathology, and gene expression array data; and tools for finding correlations between phenotype and gene expression data. All data records in MPD are discoverable through federated database systems including the Neuroinformatics Framework. All aging data generated by the JAX NSC can be found on MPD.

The Data and Statistical Core works closely with the MPD to coordinate the release of primary data. Agreements in place with pilot project awardees and collaborators ensure that these data are released to MPD in a timely manner. In addition, flat data files (.csv format) and interactive analysis tools are released on the JAX NSC website. For example, gene expression and proteomics data is the QTLViewer (https://github.com/churchill-lab/qtlweb), an interface for genetic analysis which allows easy and in-depth access to genetic mapping details.

The Data and Statistical Core engages with pilot project applicants to evaluate proposals and to make 
recommendations regarding study design, including sample size determination. Due to resource limitations, e.g., numbers of aged mice available for a study, the Core often recommends approaches to simplify and reduce the scale of proposed studies to achieve adequate power with the resources available. The Core implements project tracking and management practices in LIMS data tables and forms tailored to each study. For investigators who are using genetically diverse mouse models in aging research for the first time, the Core will guide the investigator through study design, analysis, and interpretation.

\section{Image Analysis Core}

Aging is characterized by a decline in physiologic function of all organs and increasing rates of disease and mortality. While these macro-level changes in organ function are well-documented, relatively little is known about the underlying processes that drive the loss of function and the subsequent increase in susceptibility to disease. Due to the invasive nature of biopsies, our ability to study age-related structural changes in human organs has been limited. In contrast, mouse models allow access to tissues at multiple, specific ages and under controlled conditions. The JAX NSC has already demonstrated the success of combining genetic diversity with image analysis to identify genetic loci driving mesangial matrix expansion [23], lipoprotein deposits [24], and tertiary lymphoid organ formation [25], and has been working on new methods to automatically identify and quantify histological features [26, 27].

The goal of the newly established Image Analysis Core is to develop and provide resources for the geroscience community to aid in computer-assisted histopathological analysis and discovery of age-related histological features. The Image Analysis Core advances aging research by automating an advanced image analysis system and scoring platform that can be used to discover and quantify age-related histological features. The benefits of such an automated imaging pipeline are that early detection of histological phenotypes will predict later, age-related nonpathological and pathological outcomes. An automated imaging pipeline is also useful for determining the effect of interventions in aging studies.

The NIA-funded Geropathology Research Network (GRN) has been established to enhance the translational value of geropathology for preclinical research studies in anti-aging clinical trials (https:// geropathology.org). The network consists of an interdisciplinary team of pathologists and scientists with expertise in the comparative pathobiology of aging. The GRN has established protocols and guidelines as well as a repository of well-annotated slides of tissues from aged mice, all contributing to a more uniform assessment of geropathology. The GRN has recently published the geropathology grading platform (GGP) that can be used to distinguish age-related differences regarding the absence or presence and severity of specific histological lesions [28]. Despite these resources, manual assessment in studies that involve large sample sizes or quantification for statistical analysis remains a challenge and is prone to variation due to observer bias as reported by the GRN [28]. With the development of computer image analysis and machine learning, a logical next step is to build on the work of the GRN and capture their GGP in automated pipelines in which the features of interest can be identified and quantified.

The Image Analysis Core pipeline is currently trained to identify specific features in the kidney, but is easily adaptable to other tissues. Based on conversations with investigators in the geroscience field, the heart, liver, and lung are the three most frequent tissues assessed by the pathologists of the GRN. The GGP [28] will be used as a guide in adapting the pipeline for heart, liver, and lung. Specifically, algorithms will be trained to identify and grade for lesions in the heart associated with arteriosclerosis, cardiomyopathy/myocardial fibrosis, myocardial inflammation, myxomatous change of the valve(s), and lymphoid aggregates; lung lesions associated with airway metaplasia or hyperplasia, vascular hypertrophy, pulmonary fibrosis, pneumonitis or other inflammation, and lymphoid aggregates; and liver lesions associated with hepatocellular degeneration/ necrosis, hepatic lipidosis, periportal inflammation, bile duct hyperplasia/cysts, lymphoid aggregates, and microgranulomas.

In addition to the heart, liver, and lung, investigators have indicated their interest in the development of pipelines for other tissues, including the spleen, thymus, intestine, reproductive organs, and brain. The Image Analysis Core will work with external investigators to train and validate pipelines for their tissues of interest. 
Once the pipelines have been trained and validated, they will be made available as an open source to the community. All scripts, manuals, and other materials for each pipeline will be made available in the Github repository (github.com/TheJacksonLaboratory/AgingHistology) after validation. An important step in this process is that the pipelines will be user-friendly and that training will be provided to explain the different components of the pipeline and how they work.

\section{Conclusion}

The JAX NSC provides research resources and training for geroscience investigators interested in the role of genetics and genetic diversity in fundamental process of aging and diseases of human aging using the laboratory mouse as a model system. The Center supports geroscience researchers through the provision of pilot funds and other resources and expertise to promising investigators and projects (Research Development Core) and is organized around three highly integrated research resource cores (Animal and Phenotyping, Data and Statistical, and Image Analysis Cores) that execute key initiatives of proposed projects. The JAX NSC maintains advanced, genetically diverse populations of aged mice, performs state-ofthe-art phenotyping of age-relevant traits, and provides systems genetics analysis of complex data sets. All the data generated by the JAX NSC is made publically available to the geroscience community.

Funding This work was supported by the National Institutes of Health grant to The Jackson Laboratory Nathan Shock Center of Excellence in the Basic Biology of Aging (AG038070).

\section{References}

1. Schoenmaker M, de Craen AJ, de Meijer PH, Beekman M, Blauw GJ, Slagboom PE, et al. Evidence of genetic enrichment for exceptional survival using a family approach: the Leiden Longevity Study. Eur J Hum Genet. 2006;14(1):79-84. https://doi.org/10.1038/sj.ejhg.52015 08.

2. Broer L, Buchman AS, Deelen J, Evans DS, Faul JD, Lunetta KL, et al. GWAS of longevity in CHARGE consortium confirms APOE and FOXO3 candidacy. J Gerontol A Biol Sci Med Sci. 2015;70(1):110-8. https://doi.org/ 10.1093/gerona/glu166.
3. Murabito JM, Yuan R, Lunetta KL. The search for longevity and healthy aging genes: insights from epidemiological studies and samples of long-lived individuals. J Gerontol A Biol Sci Med Sci. 2012;67(5):470-9. https://doi.org/ 10.1093/gerona/gls089.

4. Newman AB, Walter S, Lunetta KL, Garcia ME, Slagboom PE, Christensen K, et al. A meta-analysis of four genome-wide association studies of survival to age 90 years or older: the Cohorts for Heart and Aging Research in Genomic Epidemiology Consortium. J Gerontol A Biol Sci Med Sci. 2010;65(5):478-87. https://doi.org/10.1093/ gerona/glq028.

5. Timmers PR, Mounier N, Lall K, Fischer K, Ning Z, Feng $\mathrm{X}$, et al. Genomics of 1 million parent lifespans implicates novel pathways and common diseases and distinguishes survival chances. Elife. 2019;8:e39856. https://doi.org/10. 7554/eLife.39856.

6. Collaborative CC. The genome architecture of the Collaborative Cross mouse genetic reference population. Genetics. 2012;190(2):389-401. https://doi.org/10.1534/genet ics.111.132639.

7. Churchill GA, Gatti DM, Munger SC, Svenson KL. The Diversity Outbred mouse population. Mamm Genome. 2012;23(9-10):713-8. https://doi.org/10.1007/ s00335-012-9414-2.

8. Chocron ES, Munkacsy E, Pickering AM. Cause or casualty: the role of mitochondrial DNA in aging and ageassociated disease. Biochim Biophys Acta Mol Basis Dis. 2019;1865(2):285-97. https://doi.org/10.1016/j.bbadis. 2018.09.035.

9. Srivastava A, Morgan AP, Najarian ML, Sarsani VK, Sigmon JS, Shorter JR, et al. Genomes of the mouse Collaborative Cross. Genetics. 2017;206(2):537-56. https://doi. org/10.1534/genetics.116.198838.

10. Ackert-Bicknell CL, Anderson LC, Sheehan S, Hill WG, Chang B, Churchill GA, et al. Aging research using mouse models. Curr Protoc Mouse Biol. 2015;5(2):95133. https://doi.org/10.1002/9780470942390.mo140195.

11. Sukoff Rizzo SJ, Anderson LC, Green TL, McGarr T, Wells G, Winter SS. Assessing healthspan and lifespan measures in aging mice: optimization of testing protocols, replicability, and rater reliability. Curr Protoc Mouse Biol. 2018;8(2):e45. https://doi.org/10.1002/cpmo.45.

12. Mesner LD, Calabrese GM, Al-Barghouthi B, Gatti DM, Sundberg JP, Churchill GA, et al. Mouse genome-wide association and systems genetics identifies Lhfp as a regulator of bone mass. PLoS Genet. 2019;15(5):e1008123. https://doi.org/10.1371/journal.pgen.1008123.

13. Ouellette AR, Neuner SM, Dumitrescu L, Anderson LC, Gatti DM, Mahoney ER, et al. Cross-species analyses identify Dlgap2 as a regulator of age-related cognitive decline and Alzheimer's dementia. Cell Rep. 2020;32(9):108091. https://doi.org/10.1016/j.celrep.2020. 108091.

14. Yuan R, Musters CJM, Zhu Y, Evans TR, Sun Y, Chesler EJ, et al. Genetic differences and longevity-related phenotypes influence lifespan and lifespan variation in a sexspecific manner in mice. Aging Cell. 2020;19(11):e13263. https://doi.org/10.1111/acel.13263.

15 Thompson MJ, Chwialkowska K, Rubbi L, Lusis AJ, Davis RC, Srivastava A, et al. A multi-tissue full 
lifespan epigenetic clock for mice. Aging (Albany NY). 2018;10(10):2832-54. https://doi.org/10.18632/aging. 101590.

16. Bogue MA, Churchill GA, Chesler EJ. Collaborative Cross and Diversity Outbred data resources in the Mouse Phenome Database. Mamm Genome. 2015;26(9-10):51120. https://doi.org/10.1007/s00335-015-9595-6.

17. Takemon Y, Chick JM, GerdesGyuricza I, Skelly DA, Devuyst O, Gygi SP, et al. Proteomic and transcriptomic profiling reveal different aspects of aging in the kidney. Elife. 2021;10:e62585. https://doi.org/10.7554/eLife. 62585.

18. Haque S, Ames RM, Moore K, Pilling LC, Peters LL, Bandinelli $\mathrm{S}$, et al. circRNAs expressed in human peripheral blood are associated with human aging phenotypes, cellular senescence and mouse lifespan. Geroscience. 2020;42(1):183-99. https://doi.org/10.1007/ s11357-019-00120-z.

19. Yu W, Ackert-Bicknell C, Larigakis JD, MacIver B, Steers WD, Churchill GA, et al. Spontaneous voiding by mice reveals strain-specific lower urinary tract function to be a quantitative genetic trait. Am J Physiol Renal Physiol. 2014;306(11):F1296-307. https://doi.org/10.1152/ajpre nal.00074.2014.

20. Whitehead JC, Hildebrand BA, Sun M, Rockwood MR, Rose RA, Rockwood K, et al. A clinical frailty index in aging mice: comparisons with frailty index data in humans. J Gerontol A Biol Sci Med Sci. 2014;69(6):62132. https://doi.org/10.1093/gerona/glt136.

21. An JY, Kerns KA, Ouellette A, Robinson L, Morris HD, Kaczorowski C, et al. Rapamycin rejuvenates oral health in aging mice. Elife. 2020;9:e54318. https://doi.org/10. 7554/eLife.54318.

22. Bogue MA, Grubb SC, Walton DO, Philip VM, Kolishovski G, Stearns T, et al. Mouse Phenome Database: an integrative database and analysis suite for curated empirical phenotype data from laboratory mice. Nucleic Acids Res. 2018;46(D1):D843-50. https://doi.org/10.1093/nar/ gkx1082.
23. Noordmans GA, Caputo CR, Huang Y, Sheehan SM, Bulthuis M, Heeringa $\mathrm{P}$, et al. Genetic analysis of mesangial matrix expansion in aging mice and identification of Far2 as a candidate gene. J Am Soc Nephrol. 2013;24(12):1995-2001. https://doi.org/10.1681/ASN. 2012080838 .

24. Noordmans GA, Huang Y, Savage H, van Dijk MC, Schaart G, van den Bergh Weerman MA, et al. Genetic analysis of intracapillary glomerular lipoprotein deposits in aging mice. PLoS ONE. 2014;9(10):e111308. https:// doi.org/10.1371/journal.pone.0111308.

25. Huang Y, Caputo CR, Noordmans GA, Yazdani S, Monteiro LH, van den Born J, et al. Identification of novel genes associated with renal tertiary lymphoid organ formation in aging mice. PLoS ONE. 2014;9(3):e91850. https://doi.org/10.1371/journal.pone.0091850.

26. Sheehan S, Mawe S, Cianciolo RE, Korstanje R, Mahoney JM. Detection and classification of novel renal histologic phenotypes using deep neural networks. Am J Pathol. 2019;189(9):1786-96. https://doi.org/10.1016/j.ajpath. 2019.05.019.

27. Sheehan SM, Korstanje R. Automatic glomerular identification and quantification of histological phenotypes using image analysis and machine learning. Am J Physiol Renal Physiol. 2018;315(6):F1644-51. https://doi.org/10.1152/ ajprenal.00629.2017.

28. Snyder JM, Snider TA, Ciol MA, Wilkinson JE, Imai $\mathrm{DM}$, Casey KM, et al. Validation of a geropathology grading system for aging mouse studies. Geroscience. 2019;41(4):455-65. https://doi.org/10.1007/ s11357-019-00088-w.

Publisher's note Springer Nature remains neutral with regard to jurisdictional claims in published maps and institutional affiliations. 